\title{
CLASSICAL CONDITIONING IN A SIMPLE WITHDRAWAL REFLEX IN APLYSIA CALIFORNICA ${ }^{1}$
}

\author{
THOMAS J. CAREW, ${ }^{2}$ EDGAR T. WALTERS, ${ }^{3}$ AND ERIC R. KANDEL \\ Center for Neurobiology and Behavior, Departments of Physiology and Psychiatry, College of Physicians and Surgeons, Columbia \\ University, New York, New York 10032 and New York State Psychiatric Institute, New York, New York 10032
}

\begin{abstract}
The ability of Aplysia and other gastropod molluscs to exhibit complex behaviors that can be modified by associative learning has encouraged us to search for an elementary behavior controlled by a simple and well analyzed neural circuit that also can be modified by this type of learning. Toward that end, we have now produced classical conditioning in the defensive siphon and gill withdrawal reflex of Aplysia. We used as a conditioned stimulus (CS) a light tactile stimulus to the siphon, which produces weak siphon and gill withdrawal. As the unconditioned stimulus (US), we used a strong electric shock to the tail, which produces a massive withdrawal reflex. Specific temporal pairing of the CS and US endowed the CS with the ability of triggering enhanced withdrawal of both the siphon and the gill. Random or unpaired presentations of the CS and US, as well as presentations of the CS or US alone, produced either no enhancement or significantly less enhancement than paired presentations of the CS and US. The conditioning is acquired rapidly (within 15 trials) and is retained for several days. The conditioned response is abolished completely by removal of the abdominal ganglion and many of the neurons involved in the conditioning have been identified in this ganglion previously. These include the sensory neurons and several interneurons in the CS pathway and the siphon and gill motor neurons of the conditioned and unconditioned response pathways. Moreover, the sensory neurons of the US pathway have been identified in the pleural ganglia. As a result of its simplicity, it should be possible in this reflex to specify neurons that are causally related to the conditioned response. Since this reflex also exhibits nonassociative learning, it also may be possible to compare associative and nonassociative learning on a mechanistic level.
\end{abstract}

A number of molluscan preparations have been shown recently to exhibit various forms of associative learning (Mpitsos and Davis, 1973; Mpitsos and Collins, 1975; Gelperin, 1975; Crow and Alkon, 1978; Walters et al., 1979, 1981a; Davis et al., 1980; Sahley et al., 1981a; Lukowiak and Sahley, 1981). In many of these, it has been possible to record some neural correlates of the learning process (Davis and Gillette, 1978; Crow and Alkon, 1980; Chang and Gelperin, 1980; Carew et al.,

\footnotetext{
'This work was supported by fellowships from the National Institutes of Mental Health to T. J. C. (5KO2MH0081), E. T. W. (5T32MH1574), and E. R. K. (5KO5MH18558), by National Institute of Mental Health Grant 97, and by a grant from the McKnight Foundation. We thank J. Lupatkin, A. Bush, and J. Sliney for technical assistance with the experiments; Drs. V. Castellucci, R. Hawkins, I. Kupfermann, and K. Weiss for comments on the manuscript; L. Katz and $K$. Hilten for help in the preparation of the figures; and $H$. Ayers for typing.

${ }^{2}$ To whom correspondence should be addressed at the Center for Neurobiology and Behavior, Columbia University, College of Physicians and Surgeons, 630 West 168th Street, New York, NY 10032.

${ }^{3}$ Present address: Department of Physiology, University of Pittsburgh, School of Medicine, Pittsburgh, PA 15261.
}

1981a). However, in none of these preparations has it yet been possible to show a causal relationship between learning and a functional change in nerve cells, a necessary step toward analyzing the mechanisms underlying the associative learning. One major obstacle for demonstrating a causal relationship has been that the behaviors used in these studies-although simple by vertebrate standards-are nonetheless fairly complex. Their neural circuits are therefore only partially understood.

As a first step in overcoming this obstacle, we have attempted to produce associative learning in a very simple reflex, the defensive siphon and gill withdrawal reflex of Aplysia initiated by tactile stimulation of the siphon. This behavior is controlled by a numerically limited and well analyzed neural circuit in which a significant part of the reflex pathway consists of a set of monosynaptic connections between identified sensory and motor neurons (Castellucci et al., 1970; Byrne et al., 1974; Castellucci and Kandel, 1976; Hawkins, 1981a; Hawkins et al., 1981a, b; for review, see Kandel, 1976). We have found that this simple reflex can be conditioned rapidly and that the learning lasts for several days. This reflex also can be modified through nonassociative learning, the 
mechanisms of which have been well analyzed on a cellular level (Castellucci et al., 1970; Castellucci and Kandel, 1974, 1976; Klein and Kandel, 1978, 1980). Thus, in addition to being a potentially useful system for examining the cellular mechanisms of associative learning, this reflex also may prove useful for comparing associative and nonassociative learning on a mechanistic level.

Some of the work described in this paper has been reported previously in abstract form (Carew et al., 1981b).

\section{Materials and Methods}

One hundred seventy-five Aplysia californica weighing 150 to $400 \mathrm{gm}$ were used in the experiments. Animals were obtained from Pacific Bio-Marine Laboratories, Venice, CA. Two types of experimental preparations were used to measure different components of the defensive withdrawal reflex: (1) unrestrained animals were used to study the siphon withdrawal component and (2) restrained animals were used to study the gill withdrawal component.

Unrestrained preparations. The unrestrained animals were housed individually in perforated circular pans ( 28 $\mathrm{cm}$ diameter, $7.6 \mathrm{~cm}$ depth) at least $48 \mathrm{hr}$ prior to an experiment. The pans floated freely in a 200 -gallon tank of aerated artificial sea water (Instant Ocean) kept at $15^{\circ} \mathrm{C}$. At least $24 \mathrm{hr}$ prior to training, the posterior onethird of each parapodium of all animals was excised to allow full view of the siphon even when it was contracted. The conditioned stimulus (CS) consisted of a tactile stimulus delivered by a single nylon bristle from a paint brush held firmly by a hemostat. The stimulus was applied manually by inserting the bristle into the funnel of the siphon and briskly moving it directly upward a single time, thereby stimulating the inner surface of the siphon. We estimated the duration of contact with the siphon skin to be approximately $0.5 \mathrm{sec}$. The duration of siphon withdrawal was measured by an observer with a stop watch from stimulus onset until the moment that the siphon reversed direction and began to relax. The unconditioned stimulus (US) was an electrical stimulus across the tail. The stimulus was a $1.5-\mathrm{sec}$ pulse of $50 \mathrm{~mA}$ $\mathrm{AC}$ delivered by means of spanning electrodes (see Walters et al., 1979). Testing was always carried out using a blind procedure and, in some experiments, training was also blind. In many experiments, two observers scored the animals simultaneously. In some experiments, the CS and US were delivered via implanted electrodes. The electrodes consisted of insulated silver wire $0.7 \mathrm{~mm}$ coated diameter) from which insulation was removed for $5 \mathrm{~mm}$ at one end. The wire was bent into a barb and inserted into the tissue by means of a 21 gauge hypodermic needle. Pairs of wires were inserted approximately 5 to $10 \mathrm{~mm}$ apart in the siphon and 15 to $20 \mathrm{~mm}$ apart on either side of the tail. When electrical stimuli via implanted electrodes were used, the CS was $0.5 \mathrm{sec}$ and the US was $1.5 \mathrm{sec}$ in duration. Paired or unpaired stimuli were delivered by an automatic timing device (Amatniek, Columbia University).

Restrained preparations. Details of the methods are reported elsewhere (Carew et al., 1979b). Briefly, animals were restrained in a small aquarium and the parapodia and mantle were retracted with stainless steel hooks to reveal the gill. The siphon and tail were implanted with electrodes and all stimuli were delivered automatically as described above. After both CS and US stimulus values were chosen (to produce contractions similar to those seen in the intact, unrestrained animals), animals were assigned randomly to either a paired or unpaired experimental condition.

The gill withdrawal reflex was measured by means of a closed circuit television system. A continuous timing signal from a video timer (GYRR-G77) was superimposed on a video recording of the dorsal surface of the gill. Recordings were displayed on an 8-inch screen video monitor (Panasonic WV-930) driven by a videotape recorder (GYRR DAS 300) which permitted slow motion (1/7 real time) and stop frame analysis of gill contractions. The reflex amplitude was quantified by tracing a single frame outline of the gill (1) $1 \mathrm{sec}$ prior to a stimulus and (2) at the peak of contraction (determined by repeated slow motion observations). Tracings of the outline of the gill were made on plastic transparencies and then retraced onto tracing paper, which were cut out and weighed to the nearest milligram on an analytical balance. The difference in weight between the relaxed gill tracing and the tracing of the gill at the peak of a contraction provided a measure of the percentage reduction of the gill area during a contraction.

Gill contractions to the CS alone were measured before and after training with either a specifically paired or unpaired procedure (see "Results"). Following the sccond (post-training) test, animals received one of two types of surgical procedures: (1) deganglionation or (2) sham operation. The surgical procedure that any particular animal received was determined randomly. Details of the surgical procedures are reported elsewhere (Carew et al., 1979b). In deganglionated animals, an incision was made in the neck and the abdominal ganglion was removed by severing all of its peripheral nerves and the pleuroabdominal connectives; the incision then was sutured closed. In sham-operated animals, the surgical procedures were identical except that the abdominal ganglion, once visualized and retracted, was left intact. At least $1 \mathrm{hr}$ after surgery, all animals were tested with the same CS used during training. Following this test, in deganglionated animals, the CS intensity was progressively increased at 5-min intervals (in fixed increments proportional to the original CS intensity; see "Results") until a peripherally mediated contraction was observed. The CS intensity then was increased further until no further increment in the amplitude of gill contraction was observed.

Statistical analysis. Standard parametric statistical tests were used to evaluate the data. Individual comparisons were made with $t$ tests for either correlated (within group) or independent (between group) means, with minimum acceptable probability values elevated to compensate for multiple comparisons. Prior to these comparisons, a one-way analysis of variance was always performed to establish an overall significance level (Winer, 1962).

\section{Results}

Classical conditioning of the siphon withdrawal re. flex. Tactile stimulation of the siphon produces a defensive reflex consisting of the withdrawal of the siphon and 
the gill. We first examined whether the siphon component of the defensive withdrawal reflex could be classically conditioned. Using unrestrained freely moving animals, we paired a light tactile stimulus produced by a nylon bristle to the siphon (the conditioned stimulus or CS), which produced a weak siphon withdrawal, with a strong electric shock to the tail (the unconditioned stimulus or US), which produced massive siphon and gill withdrawal, and investigated whether the US could enhance the response to the CS. The experimental procedure is illustrated in Figure 1 (for details of the CS and US, see "Materials and Methods"). In most experiments, animals were first given a pre-test with the CS alone: the duration of siphon withdrawal was measured from the onset of the CS until the moment that the siphon began to reverse direction (that is, it began to relax). In the first experiment, animals were divided into two groups: paired $(N=10)$ and unpaired $(N=10)$. Fifteen training trials were given with an intertrial interval (iti) of $5 \mathrm{~min}$. On each trial, paired animals received the CS followed 0.5 sec later by the US; unpaired animals received the CS and US separated by $2.5 \mathrm{~min}$. Animals were tested (blind; see "Materials and Methods") with the CS alone within $15 \mathrm{~min}$ (range $=5$ to $15 \mathrm{~min}$ ) after training. The results of the first experiment are shown in Figure 2. Paired animals showed 3 times longer siphon withdrawal in response to the $\mathrm{CS}$ (mean $=64.7 \mathrm{sec}$ ) than unpaired animals (mean $=16.7$ sec; $p<0.005$ ). These findings suggested that the siphon withdrawal reflex of Aplysia could be conditioned.

Although the testing in the preceding experiment was carried out using a blind procedure, the training was not.
Thus, the possibility existed that experimenter bias in training contributed to the facilitation observed in paired animals. Training bias could be introduced in two ways: (1) in the delivery of the CS and (2) in the delivery of the US. We carried out two experiments to rule out these possibilities. In the first experiment, both the CS and US were delivered by means of implanted silver wire electrodes in the siphon and in the tail, respectively (see "Materials and Methods"). Two animals were trained at a time. The stimulus intensity of the CS was adjusted to produce a weak siphon withdrawal, roughly comparable in duration to that produced by the tactile stimulus in the previous experiment. The US was similarly adjusted to produce an unconditioned response comparable to that in the preceding experiment. After the parameters for the CS and US were chosen, animals were assigned randomly to receive either paired or unpaired training, after which, the experiment was run automatically by means of a programmable timer (see "Materials and Methods"). Animals received 15 trials (iti $=5 \mathrm{~min}$ ) and then were tested with the CS alone (weak siphon shock) within $15 \mathrm{~min}$ (range $=5$ to $15 \mathrm{~min}$ ) after training. The results are shown in Figure $3 A$. Paired animals $(N=7)$ again showed a 3-fold greater siphon withdrawal in response to the electrical CS $($ mean $=43 \mathrm{sec})$ than unpaired animals $(N=7$; mean $=12.3 ; p<0.01)$. Moreover, a within group comparison showed that paired animals exhibited significant enhancement of siphon withdrawal compared to their own pre-test scores $(p<0.05)$, whereas unpaired animals did not. In fact, unpaired animals showed a reduction of their mean scores after training.

In a second experiment also designed to rule out the

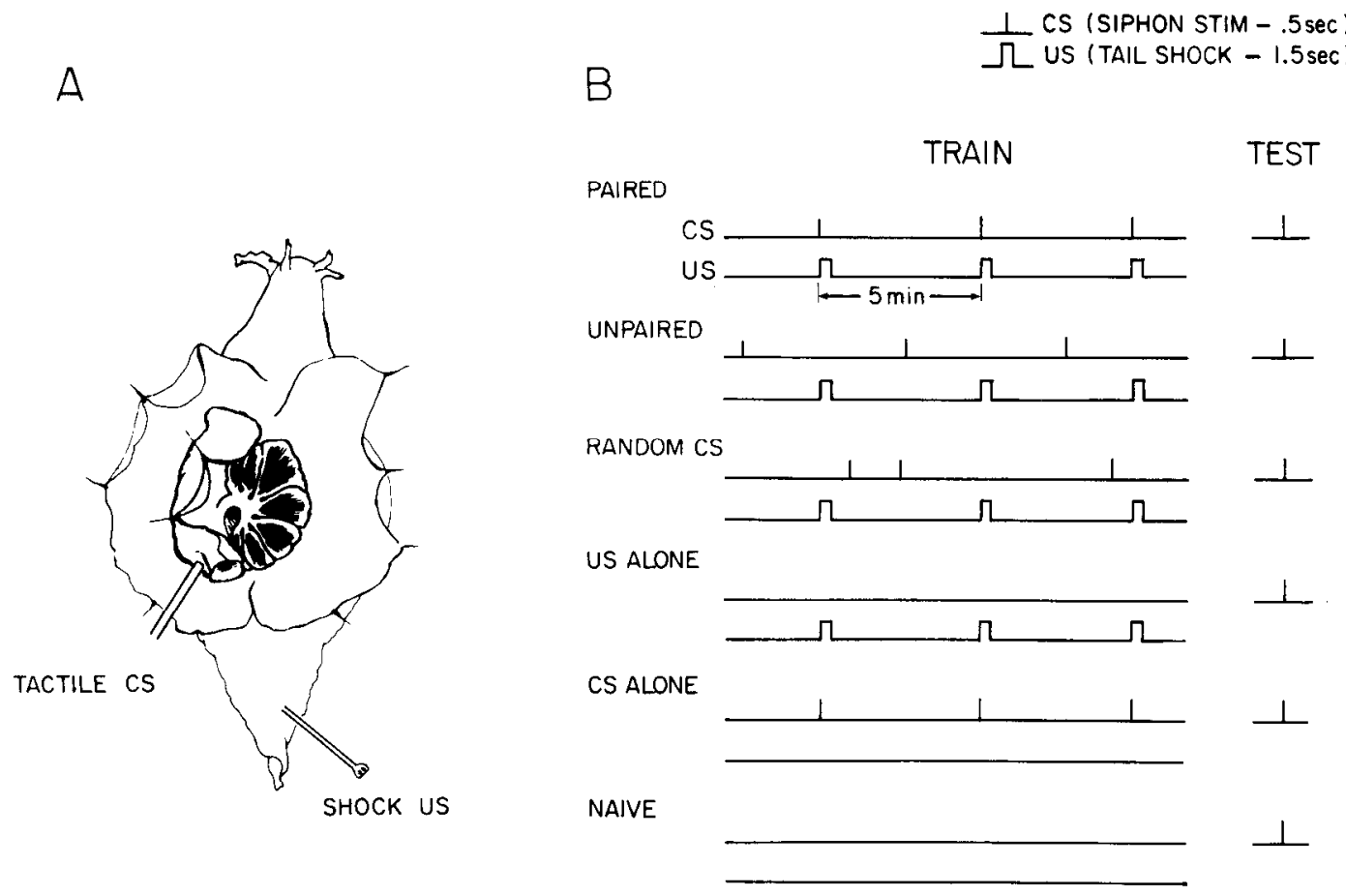

Figure 1. Classical conditioning paradigm. A, Dorsal view of Aplysia with the parapodia and mantle shelf retracted to reveal the gill. The CS was a weak tactile stimulus to the siphon delivered either by a nylon bristle or by implanted electrodes in the siphon skin. The US was a strong electrical shock to the tail delivered either by hand-held spanning electrodes or by implanted electrodes in the tail. $B$, Diagrammatic representation of the conditioning and control procedures. After training, learning was measured in the test period as the response to the CS alone. In some experiments, a pre-test with the CS alone was given prior to training in order to match groups and permit within group statistical comparisons. 
possibility of a training bias, the tactile CS (nylon bristle) used in the first experiment (Fig. 2) was used in conjunc. tion with an implanted electrical US in the tail. As in the preceding experiment, the US parameters were chosen prior to assigning animals to either a paired or unpaired condition, which was determined randomly. Animals received 15 trials (iti $=5 \mathrm{~min}$ ) and then were tested with the CS alone (nylon bristle). The results (Fig. $3 B$ ) were

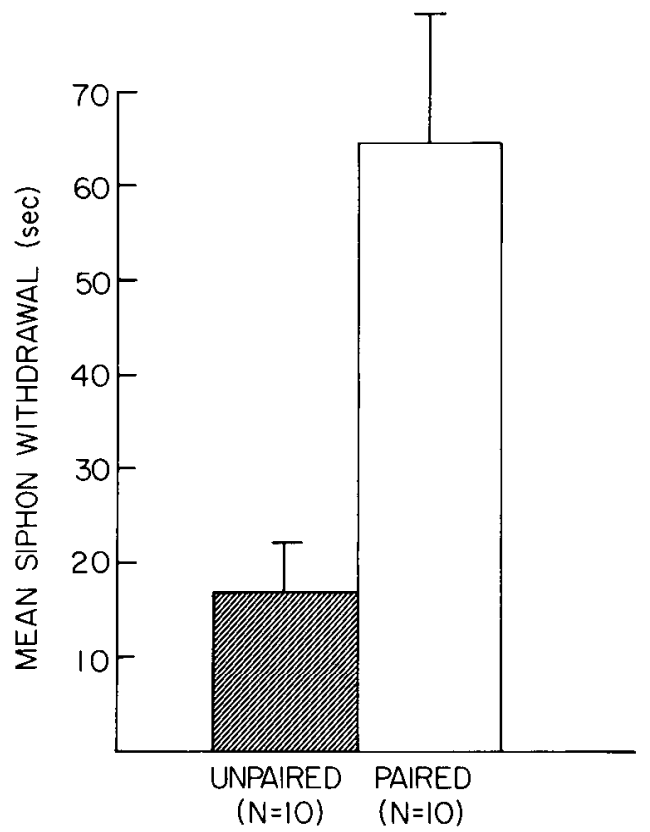

Figure 2. Classical conditioning of siphon withdrawal. Following 15 training trials (iti $=5 \mathrm{~min}$ ), animals receiving paired training exhibited significantly greater siphon withdrawal in response to the $\mathrm{CS}$ alone than animals receiving unpaired training $(p<0.005)$. The data in this and all subsequent figures are expressed as means \pm SEM.

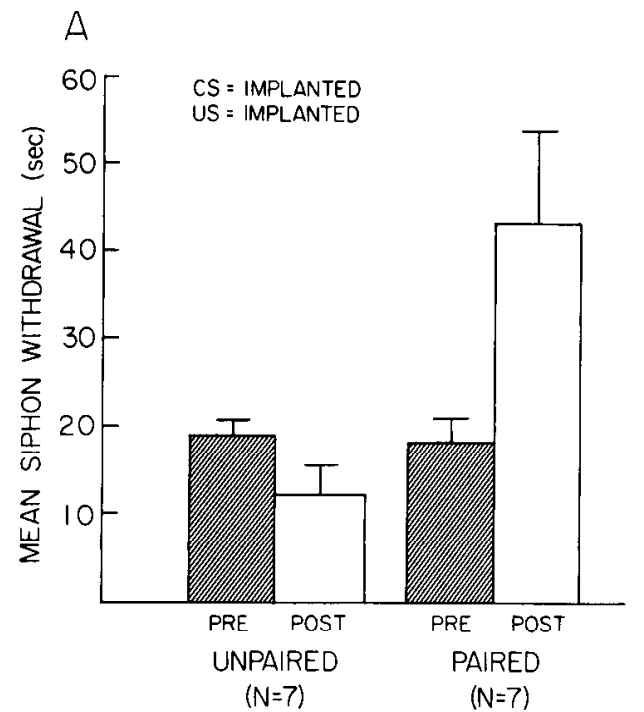

quite comparable to the previous results (Fig. 3A). Paired animals $(N=10)$ showed 4 times greater siphon withdrawal (mean $=50.2 \mathrm{sec}$ ) than unpaired animals $(N=$ 10 ; mean $=11.6 \mathrm{sec} ; p<0.005$ ). Once again, paired animals also showed significant enhancement compared to their own pre-test scores $(p<0.025)$, while unpaired animals did not. Thus, neither a training nor a testing bias appears to contribute to the facilitation observed in paired animals. Rather, the facilitation is due to the specific temporal association of the CS and US.

Acquisition and extinction of conditioned siphon withdrawal. We next examined the acquisition and extinction of the conditioned response. Six groups of animals were examined: paired $(N=9)$, unpaired $(N=9)$, random CS with respect to the US $(N=8)$, US alone $(N$ $=8)$, CS alone $(N=9)$, and untrained or naive $(N=9)$. A total of 31 training trials (iti $=5 \mathrm{~min}$ ) were given. Siphon withdrawal was tested with the CS alone (nylon bristle) immediately after the first trial and then after every 5 trials. Extinction was examined by delivering the $\mathrm{CS}$ alone for 10 trials (iti $=5 \mathrm{~min}$ ) immediately following training. Thus, the acquisition test after trial 31 served as the first extinction trial. The results are shown in Figure 4. During acquisition, siphon withdrawal in the paired group increased more than 3-fold from a mean of $8.6 \mathrm{sec}$ after trial 1 , to $32.5 \mathrm{sec}$ after trial $31\left(F_{6,48}=2.8\right.$; $p<0.05$ ). No other group exhibited significant changes during acquisition. During extinction, the paired group showed a significant decrease in siphon withdrawal ( $p$ $<0.05$ comparing trials 1 and 10). All other groups remained virtually unchanged during extinction, although there was a slight (and insignificant) tendency to show somewhat reduced responding on the last extinction trial compared to the first.

There are two interesting aspects of the results shown in Figure 4. First, the conditioned siphon withdrawal is acquired rapidly. Within group comparisons showed that

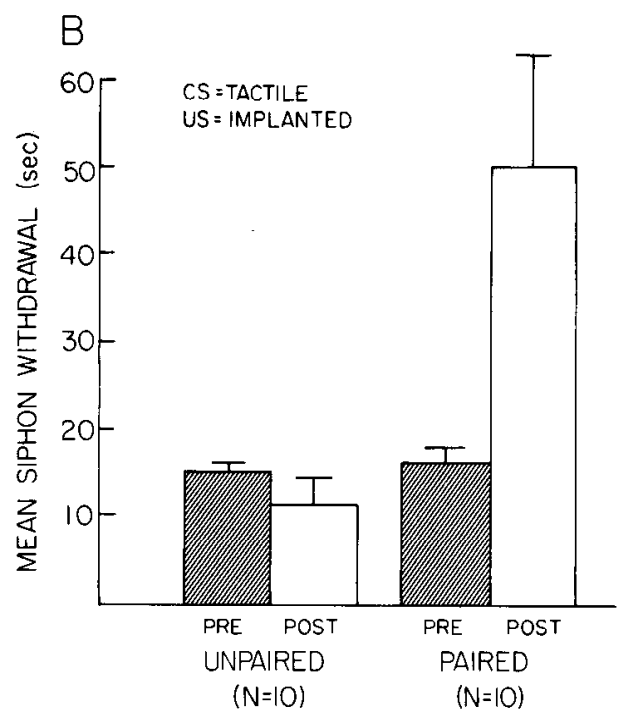

Figure 3. Controls for training bias. $A$, Both the CS and US were delivered by means of electrodes implanted in the siphon and tail, respectively (see "Materials and Methods"). After CS and US parameters were chosen, animals were assigned randomly to a paired or unpaired group and stimuli were delivered automatically. Following 15 training trials, paired animals showed significantly greater siphon withdrawal compared to unpaired animals (open bars; $p<0.01$ ) and compared to their own pre-test scores (shaded bars; $p<0.05$ ). $B$, Same experimental design as $A$ except that the CS was delivered with a nylon bristle rather than implanted electrodes. Once again, paired animals showed significantly enhanced siphon withdrawal compared both to unpaired animals (open bars; $p<0.005$ ) and to their own pre-test scores (shaded bars; $p<0.025$ ). 


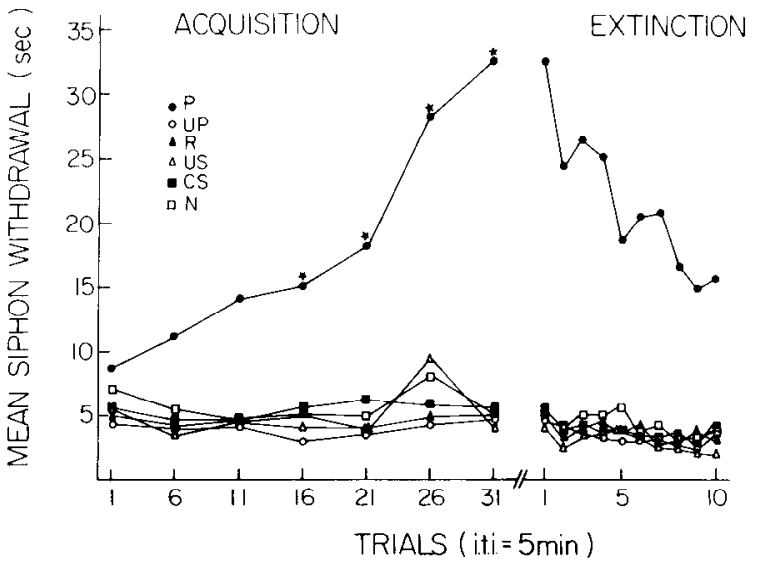

Figure 4. Acquisition and extinction of conditioned siphon withdrawal. Six groups of animals were examined: paired $(P)$, unpaired ( $U P$ ), random $(R)$, US alone (US), CS alone (CS), and untrained or naive $(N)$ (see also Fig. $1 B$ ). All groups were tested with the CS alone (nylon bristle) after the first training trial and then after every 5 trials. Following training, extinction was measured by delivering the CS alone for 10 trials (iti $=5$ min). Only the paired group showed enhanced siphon withdrawal during acquisition. A within group comparison showed that paired animals exhibited significantly enhanced siphon withdrawal compared to their own trial 1 performance in the trial 16 test and in every test thereafter (indicated by stars: trial $16, p<0.05$; trial 21, $p<0.005$; trial $26, p<0.025$; trial $31, p<$ $0.05)$. During extinction, paired animals showed a significant reduction in siphon withdrawal $(p<0.05)$, comparing trial 1 to trial 10, while all other groups remained unchanged.

paired animals exhibited significant learning (compared to their own trial 1 scores) at trial 16 and at all tests thereafter. Second, no other group except paired animals showed any facilitation during training. This was puzzling since this reflex readily shows sensitization both from head and tail shock (Pinsker et al., 1970, 1973; Carew et al., 1979a; V. F. Castellucci and R. D. Hawkins, manuscript in preparation). Why was no sensitization evident in the US alone group or in the groups that received the US and the CS in an unpaired or random manner?

A clue emerged when we tested all animals a second time $24 \mathrm{hr}$ after training (Fig. 5, open bars). The paired group again showed significantly longer siphon withdrawals (mean $=55 \mathrm{sec}$ ) than all other groups $(p<$ $0.005)$. However, now the US alone group exhibited significant sensitization $($ mean $=20.1 \mathrm{sec})$ compared to both the unpaired (mean $=9.1 \mathrm{sec})$ and random (mean $=10.5$ sec) groups ( $p<0.005$ in both cases), which, in turn, were elevated significantly compared to the groups that had no prior exposure to the US: the CS alone (mean = $5.4 \mathrm{sec}$ ) and untrained groups (mean $=6.8 \mathrm{sec}$ ). The striking difference between the scores observed immediately after training (Fig. 5, shaded bars) and $24 \mathrm{hr}$ after training (Fig. 5, open bars) suggested that the sensitization produced by the US develops more slowly than the associative effect. We therefore carried out a series of experiments to test this idea specifically.

Delayed development of sensitization. Although the results shown in Figure 5 show that sensitization is manifest within $24 \mathrm{hr}$, other studies of sensitization of this reflex suggested that sensitization should appear sooner (Pinsker et al., 1970; Carew et al., 1979a; V. F. Castellucci and R. D. Hawkins, personal communication).

To examine the possibility that sensitization develops with a delay, we carried out two experiments. In the first experiment, we gave 24 animals a pre-test with the CS alone and then delivered a single tail shock (US). The animals then were divided into three groups which were tested (with the nylon bristle) 5,30 , or 60 min after the US. Approximately $3 \mathrm{hr}$ later, the second experiment was carried out on the same animals. In this experiment, all animals received 15 trials of the US alone (iti $=5 \mathrm{~min}$ ) and then were tested 5,30 , or $60 \mathrm{~min}$ after the last US. If sensitization has a delayed onset, we would predict that animals tested soon after the US should show less sensitization of siphon withdrawal than those tested later. The results of these experiments, shown in Figure 6 , confirmed this prediction. The effects of a single US trial are shown in Figure 6A. The group of animals tested $5 \mathrm{~min}$ after the US showed no evidence of sensitization compared either to their own pre-test scores or to the groups tested 30 or 60 min later. Both of the 30- and 60min groups, on the other hand, showed significant sensitization (within group comparison: $p<0.01$ and $p<$ 0.005 , respectively). The effects of 15 trials are shown in Figure $6 B$. Once again, the group tested $5 \mathrm{~min}$ after the last US showed no significant sensitization, while the groups tested 30 or $60 \mathrm{~min}$ later exhibited significant sensitization (within group comparison: $p<0.01$ and $p$ $<0.005$, respectively). An interesting aspect of the results is that both 30 - and 60-min groups show comparable sensitization after a single US, while the 60-min group exhibits significantly greater sensitization than the 30 min group after 15 US trials, suggesting that the magnitude and temporal development of sensitization are depencient upon the number of US exposures.

The hypothesis that sensitization has a delayed onset leads to one further prediction: the 5-min group, which

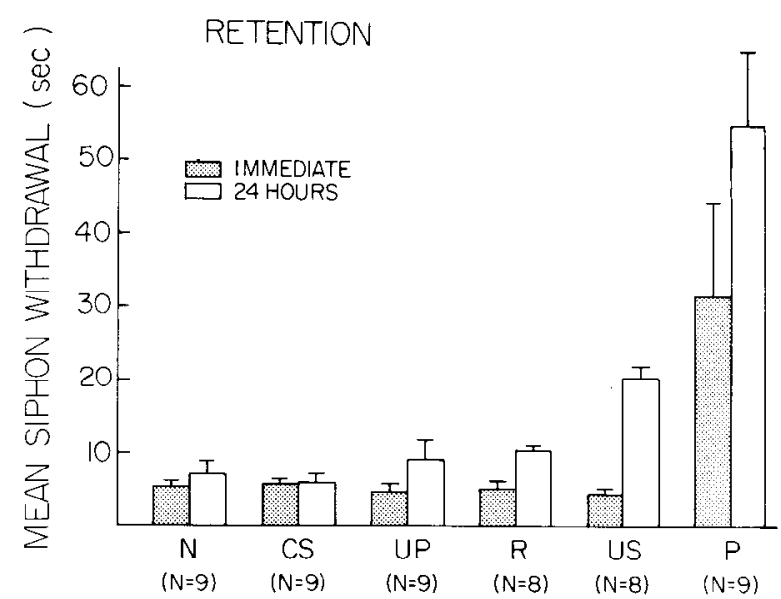

Figure 5. Comparison of immediate and 24-hr retention. All groups shown in Figure 4 were tested again $24 \mathrm{hr}$ after training. Open bars show the data from the 24-hr test; shaded bars show the scores obtained immediately after the 31st trial (Fig. 4). Paired animals were still significantly higher than all other groups $(p<0.005)$, but in the 24 -hr test, significant differences emerged among the other groups (see text for discussion). The abbreviations are defined in the legend to Figure 4. 

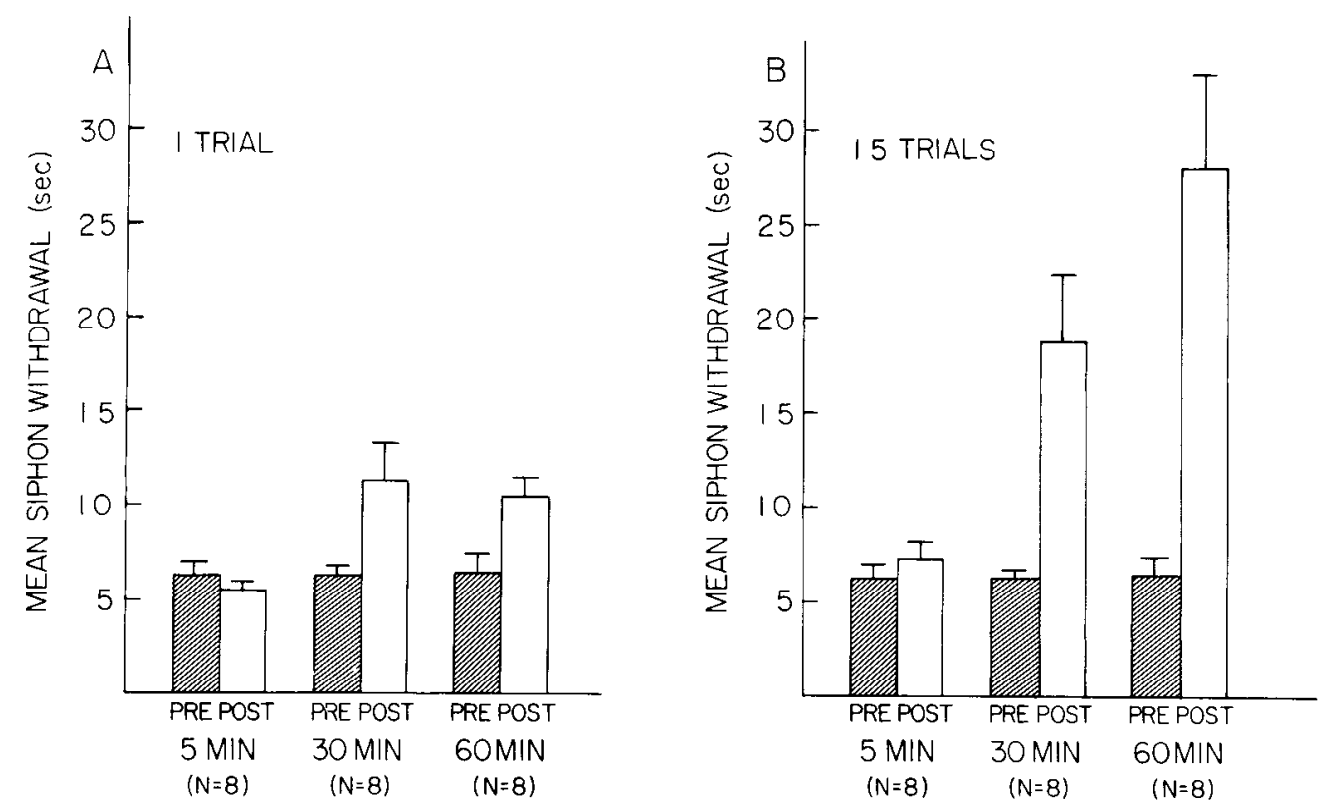

Figure 6. Delayed development of sensitization. $A$, After the presentation of a single US (tail shock), the groups were tested with the CS alone after 5, 30, or $60 \mathrm{~min}$. The 5-min group showed no sensitization compared to their own pre-test scores (shaded bars) or to the 30- and 60-min groups, which both showed significant sensitization $(p<0.01$ and $p<0.005$, respectively). $B$, The same experiment with the same groups as shown in $A$ except that 15 trials were given with the US alone ( 3 hr after the experiment shown in $A$ ). Once again, the 5 -min group showed no significant sensitization, while the 30 - and 60 -min groups did $(p<0.01$ and $p<0.005$, respectively).

showed no sensitization even after 15 trials, should show sensitization if re-tested an hour later. This prediction was confirmed. When all three groups were re-tested 1 $\mathrm{hr}$ after the 15-trial test, the 5-min group now showed significant sensitization (mean $=42.9 ; p<0.01$ compared to its previous 15 -trial test score) that was comparable to the sensitization shown by the 30 - and 60 -min groups in the 1 -hr re-test (mean $=49.4$ and 39.9 , respectively).

These results show that a tail shock produces sensitization which becomes manifest somewhat later than the associative effect. We cannot yet distinguish whether the delay in the appearance of sensitization is due to a relativcly slow time course for the development of sensitization or to a short term suppressive effect of the US which gradually wears off. It is interesting that the magnitude of the associative effect also increases after a 24hr delay (Fig. 5). This might simply reflect the fact that the associative learning also develops its maximal effect slowly but is of greater magnitude than sensitization throughout. Alternatively, sensitization may contribute to the performance of paired animals in later tests. These possibilities are not mutually exclusive; both processes could be occurring simultaneously. Moreover, the relationship of sensitization produced by tail shock, which appears to have a slight delay in onset, to sensitization produced by head shock, which has little or no delay in onset (Pinsker et al., 1970), remains to be explored.

Retention of the conditioned response. We next. examined the retention of the conditioned enhancement of siphon withdrawal. Twenty-five animals were given a pre-test with the CS alone (nylon bristle) and, on the basis of those scores, they were matched and divided into three groups: paired $(N=8)$, unpaired $(N=9)$, and US alone $(N=8)$. Following the pre-test, animals were given 30 training trials (iti $=5 \mathrm{~min}$ ). Each group then was

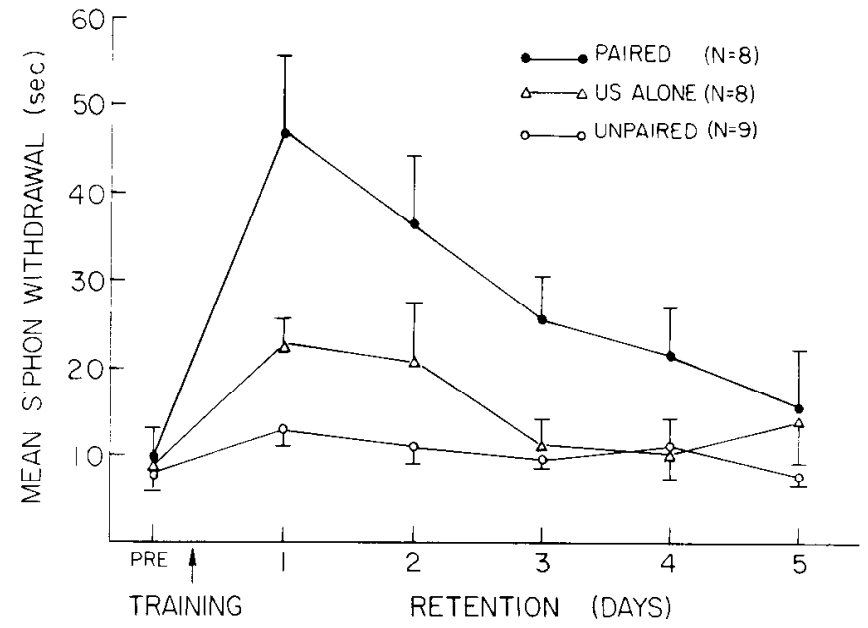

Figure 7. Retention of the conditioned response. After pretraining ( $P R E$ ), three groups were given 30 trials of paired, US alone, or unpaired training (indicated by arrow) and then were tested with the CS alone once every $24 \mathrm{hr}$. Within group comparisons showed that the paired group exhibited significant retention of the learned response up to and including day 3. The US alone group, while significantly lower than the paired group ( $p<0.01$ ), showed significant sensitization up to and including day 2. Finally, the paired group was significantly greater than both the US alone and unpaired groups on day 3 $(p<0.005$ in both cases).

tested with the CS alone at 24 -hr intervals. The results are shown in Figure 7. Consistent with previous experiments (Fig. 5), $24 \mathrm{hr}$ after training, paired animals showed significantly greater siphon withdrawal than the US alone and the unpaired groups $(p<0.01$ and $p<0.005$. respectively), and the US alone group was significantly greater than the unpaired group $(p<0.05)$. Repeated 
testing over the next 5 days showed that the paired effect gradually declines. A within group analysis showed that the paired animals exhibited significant retention of conditioning (compared to their own pre-test scores) up to and including the 3rd day of testing (see Fig. 7 legend). The sensitization control group (US alone) showed roughly similar kinetics of retention as the paired group, but the magnitude of facilitation was significantly less. A within group analysis showed that the US alone group exhibited significant sensitization up to and including the 2nd day of testing (see Fig. 7 legend). The unpaired group showed modest enhancement (which was not significantly different from their pretraining performance) only on the 1st day of testing. These results show that 30 conditioning trials (iti $=5 \mathrm{~min}$ ) can produce retention of the conditioned response that lasts for at least 3 days. Since repeated testing of the same animals over days constitutes an extinction procedure, it is possible that the time course of retention might actually be slightly longer.

Classical conditioning of the gill withdrawal reflex. Thus far, we have focused on conditioning of the siphon withdrawal component of the defensive withdrawal reflex because it can be readily examined over many days in freely moving animals. However, it is important to establish whether the gill withdrawal component of the reflex can also be conditioned, for it is this component that has been examined most extensively on a cellular level.

A direct measure of the gill withdrawal component
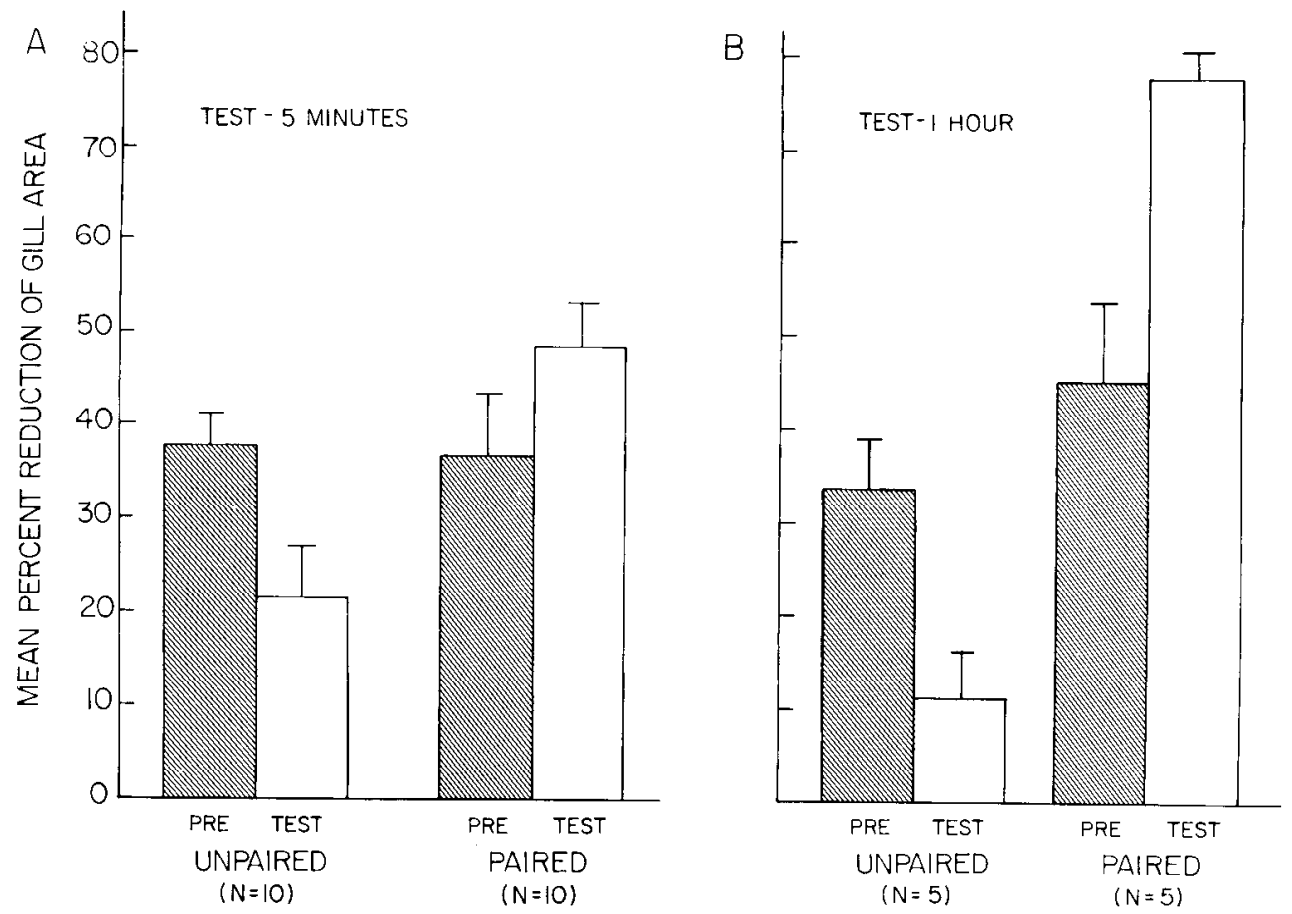

Figure 8. Classical conditioning of gill withdrawal. Animals were trained one at a time with electrodes implanted in the siphon and tail to deliver the CS and US, respectively (see text and Fig. $3 A$ ), and gill withdrawal was measured with a videotape in a restrained preparation (see "Materials and Methods"). After CS parameters were chosen and a pre-test gill withdrawal to the CS alone was measured, animals were assigned randomly to a paired or unpaired training condition. Following 15 training trials (iti $=5 \mathrm{~min}$ ), animals again were tested with the CS alone. $A$, When tested 5 min after training, the paired group showed significantly greater gill withdrawal than the unpaired group (open bars; $p<0.005$ ). Unpaired animals showed a significant reduction of gill withdrawal compared to their own pre-test scores (shaded bars; $p<0.025$ ), while paired animals showed an increase over their pre-test scores. This increase, however, was not statistically significant. $B$, A subset $(N=5)$ of both paired and unpaired animals in $A$ were tested again $1 \mathrm{hr}$ later (see text and Fig. $10 \mathrm{~A}$ ). Paired animals still showed significantly greater gill withdrawal than unpaired animals $(p<0.005)$ but, in addition, now showed significantly greater withdrawal scores compared to their own pre-test performance $(p<0.05)$. 
received the US at CS offset; unpaired animals received the CS and US separated by $2.5 \mathrm{~min}$. Five minutes after training, animals again were tested with the CS alone (Fig. $8 A$ ). Paired animals $(N=10)$ showed a significant enhancement of gill withdrawal compared to unpaired animals $(N=10 ; p<0.005)$. In fact, unpaired animals showed a significant reduction in gill withdrawal $(p<$ $0.025)$ compared to their own pretraining scores. Paired animals showed an increase following training, but this increase did not reach statistical significance when compared to their own pretraining scores and thus appeared to differ from conditioned siphon withdrawal, where significant increases over pretraining scores were observed (for example, see Fig. 3). However, a randomly selected subset of the paired group $(N=5)$ and of the unpaired group $(N=5)$ were re-tested $1 \mathrm{hr}$ later (as will be discussed in the following section; see also Fig. 10A) and, in that test, they showed a significant increase not only compared to unpaired animals $(p<0.005)$ but also compared to their own pre-test scores $(p<0.05$; Fig. $8 B)$. Thus, the associative effect appears to develop somewhat more slowly for gill withdrawal compared to siphon withdrawal, perhaps due to the necessity of restraining animals to measure gill withdrawal. However, our results clearly show that the same procedures that produce classical conditioning of siphon withdrawal also produce classical conditioning of gill withdrawal.

Contribution of the central nervous system to conditioned gill withdrawal. The main goal of these studies is to produce associative learning in a behavior controlled by a simple and well analyzed neural circuit. Although gill withdrawal produced by tactile stimulation of the siphon is predominantly mediated by neural circuits in the central nervous system (Kupfermann et al., 1971, 1974; Carew et al., 1979b), with certain types of tactile stimuli, the peripheral nervous system can also make a contribution (Peretz et al., 1976; Carew et al., 1979b). Therefore, it is important to specify the degree to which the abdominal ganglion mediates classical conditioning of gill withdrawal. To examine this question, we first conditioned the animals and then studied the effects of surgical removal of the abdominal ganglion, which disconnects the mantle organs from the central nervous system. The paired and unpaired animals in the preceding experiment (Fig. 8) were used in the study. Following the post-training test (open bars in Fig. 8), paired and unpaired animals were subdivided randomly into two groups: the deganglionated group ( $N=10: 5$ paired and 5 unpaired) had their abdominal ganglion surgically removed; the shom-operated group $(N=10: 5$ paired and 5 unpaired) received identical surgical procedures except that the abdominal ganglion was left intact (for details, see "Materials and Methods"). At least $1 \mathrm{hr}$ after surgery, all animals were tested with the identical CS with which they had been trained. An example of the results in paired animals is shown in Figure 9. A summary of the results of all 20 experiments is shown in Figure 10. Both paired and unpaired groups showed a profound and significant reduction of reflex response to the CS following deganglionation compared both to their own pre-test scores $(p<0.005$ in both cases) and to their post-conditioning test scores $(p<0.005$ in both cases; Fig. 10B). Paired deganglionated animals showed a gill withdrawal
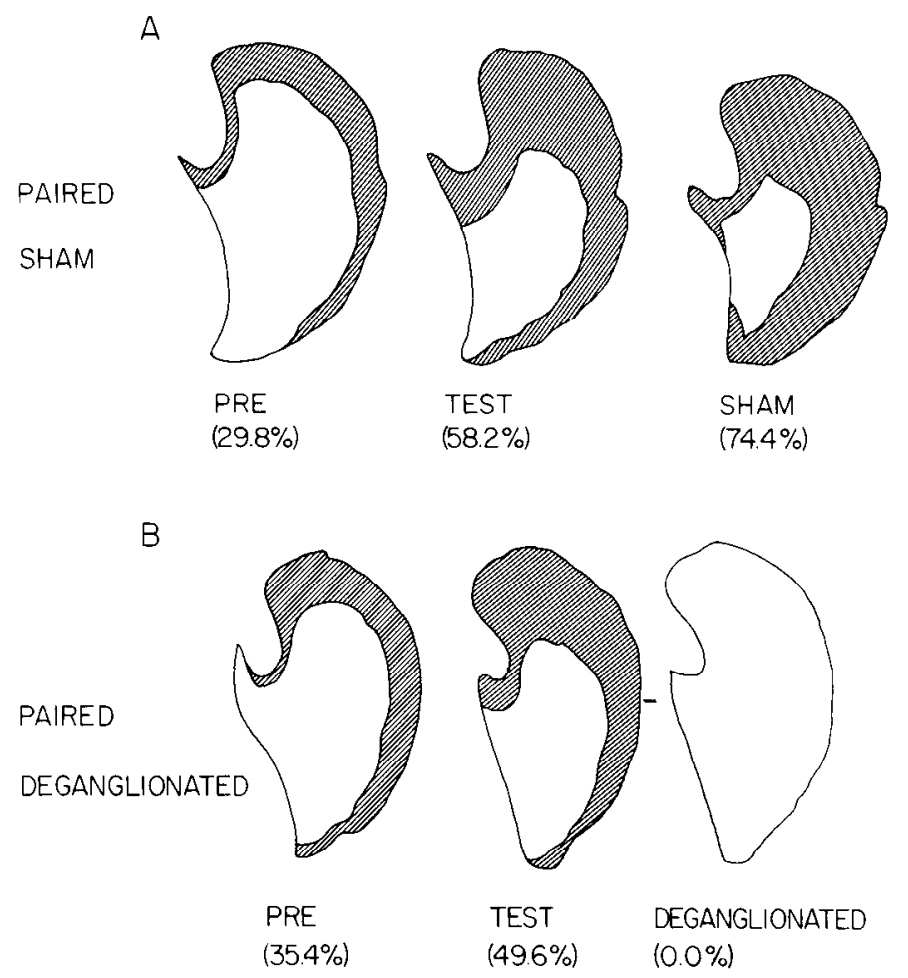

Figure 9. Examples of the effects of either sham operation or removal of the abdominal ganglion on conditioned gill withdrawal. The data are tracings of the outline of the gill (1) immediately prior to the CS in the relaxed state and (2) at the peak of the CS-evoked contraction. These two traces have been superimposed, with the shaded area representing a quantitative measure of the amplitude of the contraction in terms of the reduction in the gill area. The percentages in parentheses are the percentage reduction in the gill area computed with the formula (relaxed peak/relaxed) $\times 100$. A, After a pre-test $(P R E)$ which produced a $29.8 \%$ reduction in the gill area, the animal received paired training and then was tested with the CS alone (TEST), which produced a $58.2 \%$ contraction. One hour following a sham operation (see text and "Materials and Methods"), the response ( $S H A M$ ) is even larger, $74.4 \%$. $B$, The same procedure was followed as in $A$ except that the abdominal ganglion was removed after the test. This paired animal also showed conditioning ( $P R E=35.4 \% ; T E S T=49.6 \%)$. However, $1 \mathrm{hr}$ after deganglionation, the conditioned response is abolished; the CS alone produces no contraction.

that was reduced to a mean of $0.4 \%$ (compared to a mean pre-test score of $28.7 \%$ ) and unpaired animals showed a reduction to a mean of $9.06 \%$ (compared to a mean pretest score of $41.9 \%$ ).

The sham-operated groups showed dramatically different results than the deganglionated groups. Unpaired sham-operated animals showed no significant change in performance following surgery, comparing test scores to post-test scores (Fig. 10A). Paired sham-operated animals, rather than showing the significant decrease observed in deganglionated animals, showed a significant increase compared to both their own pre-test scores and test scores ( $p<0.05$ in both cases; Fig. 10A). The fact that paired animals exhibit a significantly larger associative effect in the post-surgery test shows two important points: (1) the surgical procedures per se do not disrupt the conditioning. Thus, the abolition of the conditioned response in deganglionated animals (Fig. 10B) is due to 
removal of the central nervous system; (2) as in the freely moving animal, the associative effect appears to increase in magnitude over time after training. Thus, although paired animals show significantly higher scores compared to unpaired animals when tested immediately after train-
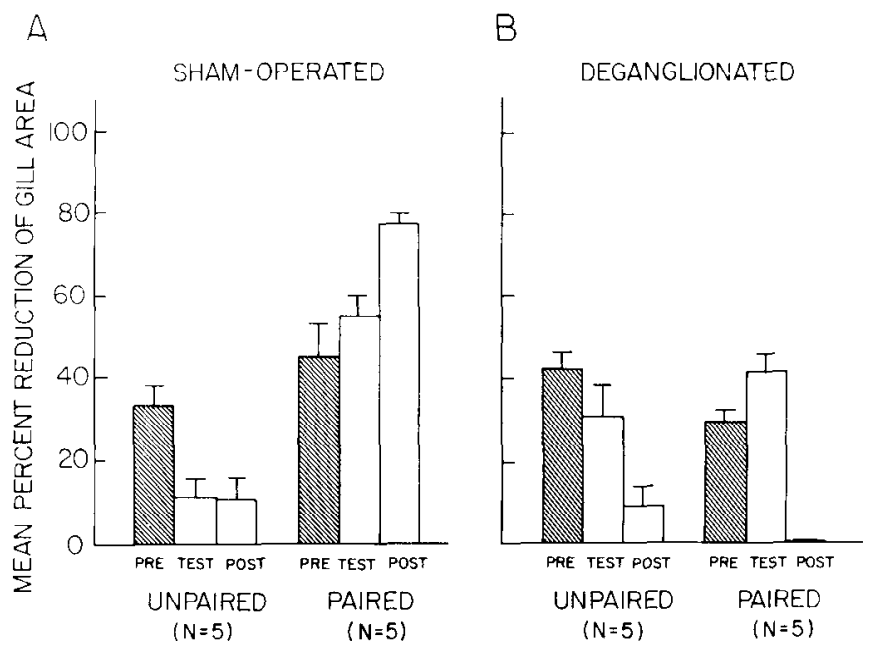

Figure 10. Contribution of the central nervous system to conditioned gill withdrawal. Paired and unpaired animals shown in Figure $8 A$ were divided into two subgroups: one received a sham operation; the other had their abdominal ganglion removed (see text and "Materials and Methods"). One hour after surgery, all animals were tested again (POST). $A$, Sham-operated unpaired animals show no change in performance after surgery (compare POST to TEST), while shamoperated paired animals showed a significant increase in performance $(p<0.05) . B$, Both unpaired and paired deganglionated animals show a significant reduction in gill withdrawal after surgery (comparing POST to TEST, $p<0.005$ in both cases). The abolition of the conditioned response in the paired animals is due to the removal of the central nervous system rather than to surgery per se, since sham-operated paired animals show a significant increase in performance.

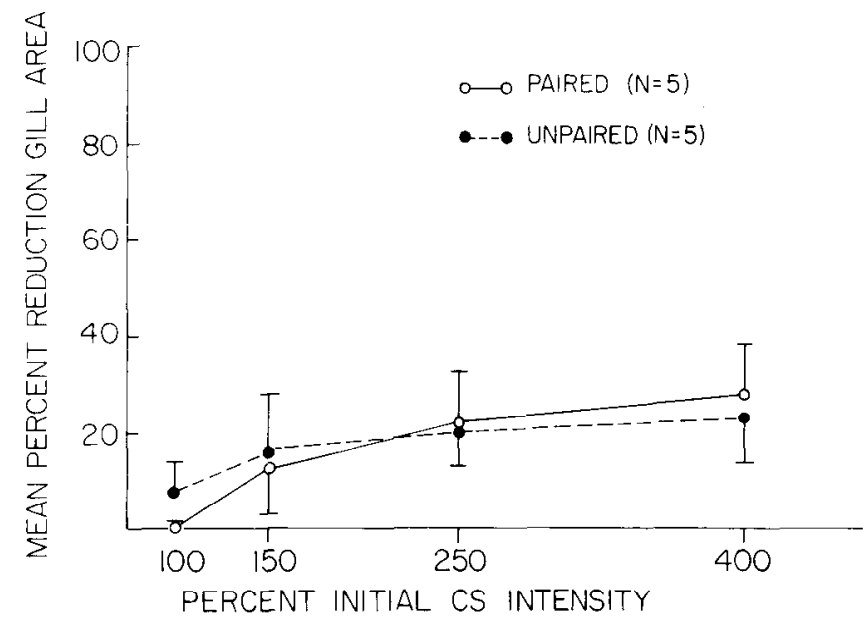

Figure 11. Input-output curve for peripherally mediated gill contractions in paired and unpaired animals. After the postsurgical test (Fig. $10 B$ ), the peripheral nervous system was intentionally recruited in paired and unpaired animals by progressively increasing the CS intensity (as a fixed percentage of the CS intensity with which the animal was trained). There was no significant difference between paired and unpaired animals in their threshold for contraction or in the slope of their respective input-output curves. ing (Fig. $8 A$ ), even in this small sample $(N=5)$, they show significantly higher scores compared to their own pretraining scores when tested at least an hour after training (Figs. $8 B$ and $10 A$ ). The unchanged small postsurgical responses of the unpaired, sham-operated group show that the enhanced post-surgical responses in the paired (sham-operated) group are not due to sensitization resulting from the surgical procedures but rather to the retention of the conditioned response.

The results suggest that the conditioning of the gill withdrawal reflex is centrally mediated. When both paired and unpaired deganglionated animals are considered together, only an average of 5\% of the reflex remained after removal of the abdominal ganglion. Nonetheless, the possibility existed that, in these experiments, some conditioning might reside in the small peripheral component of the reflex which might be too weak to measure accurately. It has been shown previously that it is possible to recruit a greater peripheral contribution to gill withdrawal by increasing the intensity of the tactile stimulus to the siphon (Kupfermann et al., 1971; Peretz et al., 1976). We therefore intentionally recruited a greater peripheral component by progressively increasing the intensity of the CS (in fixed increments proportional to the CS intensity used during training) to determine whether any differences could be detected between paired and unpaired deganglionated animals either in the threshold intensity or in the slope of an input-output curve for peripherally mediated gill contractions. If any part of the conditioning was mediated by the peripheral nervous system, one would predict that paired deganglionated animals should have either a lower threshold or steeper input-output curve (or both) compared to unpaired deganglionated animals. The results are shown in Figure 11. We found no significant difference between paired and unpaired animals either in the threshold or in the shape of the input-output curve for peripherally mediated gill contractions.

\section{Discussion}

Classical conditioning of gill and siphon withdrawal in Aplysia. The main goal of this study was to determine whether it is possible to produce associative learning in an elementary reflex controlled by a simple and well defined neural circuit. The gill and siphon withdrawal reflex of Aplysia was a good candidate behavior because it has been shown to be capable of a high degree of behavioral plasticity: both short and long term forms of nonassociative learning, such as habituation and sensitization, are readily established (Pinsker et al., 1970, 1973; Carew et al., 1972). However, several previous studies exploring the possibility of associative learning in this reflex failed (Pinsker et al., 1973; E. T. Walters, T. J. Carew, and E. R. Kandel, unpublished observations). A critical observation leading to the present experiments was the finding that the tail of Aplysia provides a powerful input pathway for gill and siphon withdrawal (Carew et al., 1981a). This finding suggested to us that the tail might be well suited for an unconditioned stimulus (US) pathway.

Our experiments show that the repeated pairing of a light tactile conditioned stimulus (CS) to the siphon, which alone elicits a very modest gill and siphon with- 
drawal reflex, with a strong tail shock US endows the CS with the ability of triggering a significantly enhanced withdrawal of both the gill and siphon. This enhancement is acquired rapidly (within 15 trials), lasts for several days, and is associative. Presentation of the US alone as well as random or unpaired presentations of the CS and US produce significantly less facilitation than the paired presentation of the CS and US. However, although paired training produces significantly greater facilitation than all other forms of training, our data suggest that, similar to vertebrates, where certain "control" procedures actually produce different types of associative learning (Rescorla, 1967, 1978), other "control" training procedures in Aplysia are not ineffective but actually produce different forms of nonassociative learning. Thus, our data are consistent with the following interpretation: (1) the CS alone and untrained groups show the lowest responses because they are not sensitized at all (having never experienced the US) and, in the case of the CS alone, they may be partially habituated to the CS; (2) the random and unpaired groups are also partially habituated by exposure to the CS but, in addition, are sensitized by exposure to the US; thus, they are intermediate between the CS alone and untrained groups on the one hand and the US alone group on the other; (3) the US alone group is significantly higher than all the former groups because it has had no habituation due to CS exposure; thus, this group exhibits "pure" sensitization; (4) the paired group exhibits the greatest enhancement of all because the US, when paired with the CS, adds to its efficacy in a nonlinear and temporally specific way. This interpretation, although consistent with all our observations, is admittedly speculative, but it has two merits. First, it provides a unifying explanation which utilizes simple forms of nonassociative learning known to exist in this reflex. Second, it provides several testable hypotheses concerning the relationship between habituation, sensitization, and classical conditioning.

Associative learning in gastropod molluscs. It is now clear that gastropod molluscs are able to learn a variety of associative tasks. The earlier work in this area focused on whether these invertebrates could, in fact, learn an associative task (Mpitsos and Davis, 1973; Mpitsos and Collins, 1975; Gelperin, 1975; Crow and Alkon, 1978; Walters et al., 1979). More recently, the emphasis has moved in two main directions. One direction has been to explore higher order features of associative learning in gastropods and to determine the degree to which they resemble features of learning in mammals (Davis et al., 1980; Sahley et al., 1981b; Walters et al., 1981a). The other direction has been to try to simplify the associative paradigms so that they could be translated into cellular studies. In several cases, reliable neural correlates of associative learning have now been identified (Davis and Gillette, 1978; Crow and Alkon, 1980; Chang and Gelperin, 1980; Carew et al., 1981a). Moreover, in Hermissenda, important steps have been made in specifying a possible neuronal site and biophysical mechanism which may contribute to associative learning (Alkon, 1979, 1981).

The neural circuit mediating conditioned siphon and gill withdrawal. Since the ultimate goal of studying associative learning in gastropod molluscs is to analyze the cellular mechanisms of learning, it is advantageous to have the learning in a behavior whose neural circuit is well understood. The known elements in the CS and US pathways are summarized in Figure 12. Identified primary mechanoreceptor sensory neurons from the siphon, which are activated both by tactile and electrical stimulation of the skin, are known to make monosynaptic excitatory connections onto identified gill and siphon motor neurons as well as onto identified interneurons (Castellucci et al., 1970; Byrne et al., 1974; Castellucci and Kandel, 1976; Hawkins et al., 1981a). Thus, elements in the CS pathway and the conditioned and unconditioned motor pathways have been identified. More recently, we have found that the tail is a powerful input pathway for gill and siphon withdrawal (Carew et al., 1981a) and that primary sensory neurons which mediate this input are located in the pleural ganglion (Walters et al., 1981b). Thus, elements in the US pathway also have been identified. Finally, Hawkins (1982a, b) has found recently that the tail input, in addition to activating gill and siphon motor neurons, also powerfully activates identified facilitatory interneurons (the L29 cells) in the abdominal ganglion. These interneurons are known to produce presynaptic facilitation of sensory input from the siphon, which is the cellular mechanism of sensitization in the gill withdrawal reflex (Hawkins et al., 1981b). Thus, neurons have been identified in pathways for the $\mathrm{CS}$, the US, and both the conditioned and unconditioned responses.

Recently, a report has appeared of another instance of associative learning in Aplysia which involves different reflex pathways to the mantle organs. Lukowiak and Sahley (1981) reported that, when a photic stimulus (CS) to the siphon, which does not produce a gill contraction, is paired 60 to 80 times with a strong tactile stimulus directly to the gill (US), it results in the CS acquiring the ability to produce a gill contraction. Several control groups showed no gill contraction to the CS after training. Complete extinction of the paired effect was produced in three trials. The pathways mediating both the CS and

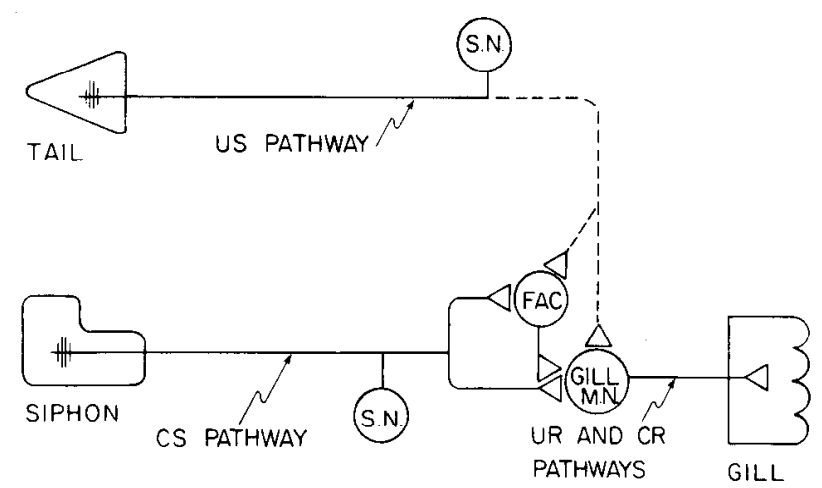

Figure 12. Schematic wiring diagram of the neural circuits involved in conditioned gill and siphon withdrawal. Neural elements have been identified previously in the primary afferent pathways (S.N., sensory neuron) for the conditioned and unconditioned stimuli ( $C S$ and US pathways) as well as in pathways that mediate the conditioned and unconditioned responses ( $C R$ and $U R$ pathways; see text). In addition, the US pathway connects to identified facilitatory interneurons $(F A C)$ and gill motor neurons ( $G I L L M . N$.) by means of one or more as yet unidentified interneurons (dashed lines). 
the US used in this learning paradigm have been shown previously to have a significant contribution from the peripheral nervous system (Lukowiak and Jacklet, 1972; Newby, 1973; Peretz, 1970; Kupfermann et al., 1971). However, insofar as the central nervous system contributes significantly to this form of learning, it may prove to be a useful preparation for a cellular analysis since the learning can be produced in a surgically reduced, in vitro preparation.

The relationship between nonassociative and associative learning in Aplysia. The same reflex that we have found here to be capable of classical conditioning has been shown previously to be capable of both short and long term forms of habituation and sensitization (Pinsker et al., 1970, 1973; Carew et al., 1972). Of particular interest for future work is the relationship between sensitization and classical conditioning. Sensitization resembles classical conditioning in that it involves the enhancement of one set of responses by activity in another pathway; it differs from classical conditioning, however, in that the temporal requirement for activity in the two pathways is much less stringent for sensitization than for classical conditioning. Because of these similarities, sensitization has been thought to constitute an elementary building block from which classical conditioning might be constructed (Kandel and Tauc, 1965; Wells, 1968; Kandel and Spencer, 1968; Razran, 1971). However, the exact relationship between these forms of learning can be resolved only by analyzing their cellular mechanisms. Since the cellular mechanisms of sensitization in this reflex are now relatively well understood (Klein and Kandel, 1978; for review, see Kandel and Schwartz, 1982), an analysis of classical conditioning can address the larger theoretical question: does associative learning employ the same class of cellular mechanisms found in sensitization?

Aplysia has been shown recently to be capable of a more complex form of associative learning resembling conditioned "fear" in vertebrates (Walters et al., 1979, 1981a). Thus, Aplysia exhibits a spectrum of learning capabilities which range from simple nonassociative processes and simple direct conditioned responses to a more complex conditioned internal state (conditioned defensive arousal), which is capable of modulating most of the animals' behavioral repertoire. It may be possible to analyze and compare each of these forms of learning in cellular terms to determine whether some cellular mechanisms are in common to several forms of learning or whether more complex forms of learning require additional or novel mechanisms.

\section{References}

Alkon, D. L. (1979) Voltage-dependent calcium and potassium ion conductances: A contingency mechanism for an associative learning model. Science 205: 810-816.

Alkon, D. L. (1980) Membrane depolarization accumulates during acquisition of an associative behavioral change. Science 210: 1375-1376

Byrne, J., V. F. Castellucci, and E. R. Kandel (1974) Receptive fields and response properties of mechanoreceptor neurons innervating siphon skin and mantle shelf in Aplysia. J. Neurophysiol. 37: 1041-1064.

Carew, T. J., V. F. Castellucci, and E. R. Kandel (1971) An analysis of dishabituation and sensitization of the gill-withdrawal reflex in Aplysia. Int. J. Neurosci. 2: 79-98.
Carew, T. J., H. M. Pinsker, and E. R. Kandel (1972) Long-term habituation of a defensive withdrawal reflex in Aplysia. Science 175: 451-454.

Carew, T. J., V. F. Castellucci, and E. R. Kandel (1979a) Sensitization in Aplysia: Rapid restoration of transmission in synapses inactivated by long-term habituation. Science 205 : 417-419.

Carew, T. J., V. F. Castellucci, J. H. Byrne, and E. R. Kandel (1979b) A quantitative analysis of the relative contribution of central and peripheral neurons to the gill-withdrawal reflex in Aplysia. J. Neurophysiol. 42: 497-509.

Carew, T. J., E. T. Walters, and E. R. Kandel (1981a) Associative learning in Aplysia: Cellular correlates supporting a conditioned fear hypothesis. Science 211: 501-504.

Carew, T. J., E. T. Walters, and E. R. Kandel (1981b) Associative learning in a simple reflex of Aplysia. Soc. Neurosci. Abstr. 7: 353.

Castellucci, V. F., and E. R. Kandel (1974) A quantal analysis of the synaptic depression underlying habituation of the gillwithdrawal reflex in Aplysia. Proc. Natl. Acad. Sci. U. S. A. 71: 5004-5008.

Castellucci, V. F., and E. R. Kandel (1976) Presynaptic facilitation as a mechanism for behavioral sensitization in Aplysia. Science 194: 1176-1178.

Castellucci, V. F., H. Pinsker, H. I. Kupfermann, and E. R. Kandel (1970) Neuronal mechanisms of habituation and dishabituation of the gill-withdrawal reflex in Aplysia. Science 167: 1745-1748.

Chang, J. J., and A. Gelperin (1980) Rapid taste aversion learning by an isolated molluscan central nervous system. Proc. Natl. Acad. Sci. U. S. A. 77: 6204-6206.

Crow, T., and D. L. Alkon (1978) Retention of an associative behavioral change in Hermissenda. Science 201: 1239-1241.

Crow, T. J., and D. L. Alkon (1980) Associative behavioral modification in Hermissenda: Cellular correlates. Science 209: 412-414.

Davis, W. J., and R. Gillette (1978) Neural correlate of behavioral plasticity in command neurons of Pleurobranchaea. Science 199: 801-804.

Davis, W. J., J. Villet, D. Lee, M. Rigler, R. Gillette, and E. Prince (1980) Selective and differential avoidance learning in the feeding and withdrawal behavior of Pleurobranchaea californica. J. Comp. Physiol. A Sens. Neural Behav. Physiol. 138: 157-165.

Gelperin, A. (1975) Rapid food-aversion learning by a terrestrial mollusk. Science 189: 567-570.

Hawkins, R. D. (1981a) Interneurons involved in mediation and modulation of the gill-withdrawal reflex in Aplysia. III. Identified facilitating neurons increase the $\mathrm{Ca}^{++}$current in sensory neurons. J. Neurophysiol. 45: 327-329.

Hawkins, R. D. (1981b) Identified facilitating neurons are excited by cutaneous stimuli used in sensitization and classical conditioning of Aplysia. Soc. Neurosci. Abstr. 7: 354.

Hawkins, R. D., V. F. Castellucci, and E. R. Kandel (1981a) Interneurons involved in mediation and modulation of the gill-withdrawal reflex in Aplysia. I. Identification and characterization. J. Neurophysiol. 45: 304-314.

Hawkins, R. D., V. F. Castellucci, and E. R. Kandel (1981b) Interneurons involved in mediation and modulation of the gill-withdrawal reflex in Aplysia. II. Identified neurons produce heterosynaptic facilitation contributing to behavioral sensitization. J. Neurophysiol. 45: 315-326.

Kandel, E. R. (1976) Cellular Basis of Behavior, Freeman Publications, San Francisco.

Kandel, E. R., and J. H. Schwartz (1982) Molecular biology of an elementary form of learning: Modulation of transmitter release through cAMP-dependent protein kinase. Science, in press.

Kandel, E. R., and W. A. Spencer (1968) Cellular neurophysi- 
ological approaches in the study of learning. Physiol. Rev. 48: 65-134.

Kandel, E. R., and L. Tauc (1965) Heterosynaptic facilitation in neurones of the abdominal ganglion of Aplysia depilans. J. Physiol. (Lond.) 181: 1-27.

Klein, M., and E. R. Kandel (1978) Presynaptic modulation of voltage-dependent $\mathrm{Ca}^{++}$current: Mechanism for behavioral sensitization in Aplysia californica. Proc. Natl. Acad. Sci. U. S. A. 75: 3512-3516.

Klein, M., and E. R. Kandel (1980) Mechanism of calcium current modulation underlying presynaptic facilitation and behavioral sensitization in Aplysia. Proc. Natl. Acad. Sci. U. S. A. 77: 6912-6916.

Kupfermann, I., H. Pinsker, V. F. Castellucci, and E. R. Kandel (1971) Central and peripheral control of gill movements in Aplysia. Science 174: 1252-1256.

Kupfermann, I., T. J. Carew, and E. R. Kandel (1974) Local, reflexive and central commands controlling gill and siphon movements in Aplysia californica. J. Neurophysiol. 37: 9901019.

Lukowiak, K., and J. W. Jacklet (1972) Habituation and dishabituation: Interactions between peripheral and central nervous systems in Aplysia. Science 178: 1306-1308.

Lukowiak, K., and C. Sahley (1981) The in vitro classical conditioning of the gill withdrawal reflex of Aplysia califor. nica. Science 212: 1516-1518.

Mpitsos, G. J., and S. D. Collins (1975) Learning: Rapid aversion conditioning in the gastropod mollusc Pleurobranchaea. Science 188: 954-957.

Mpitsos, G. J., and W. J. Davis (1973) Learning: Classical and avoidance conditioning in the mollusc Pleurobranchaea. Science 180: 317-320.

Newby, N. A. (1973) Habituation to light and spontaneous activity in the isolated siphon of Aplysia: Pharmacological observations. Comp. Gen. Pharmacol. 4: 91-100.

Peretz, B. (1970) Habituation and dishabituation in the absence of the central nervous system. Science 169: 379-381.
Peretz, B., J. W. Jacklet, and K. Lukowiak (1976) Habituation of reflexes in Aplysia: Contribution of the peripheral and central nervous systems. Science 191: 396-399.

Pinsker, H., I. Kupfermann, V. F. Castellucci, and E. R. Kandel (1970) Habituation and dishabituation of the gill-withdrawal reflex in Aplysia. Science 167: 1740-1742.

Pinsker, H. M., W. A. Hening, T. J. Carew, and E. R. Kandel (1973) Long-term sensitization of a defensive withdrawal reflex in Aplysia. Science 182: 1039-1042.

Razran, G. (1971) Mind in Evolution: An East-West Synthesis of Learned Behavior and Cognition, Houghton Mifflin, Boston.

Rescorla, R. A. (1967) Pavlovian conditioning and its proper control procedures. Psychol. Rev. 74: 71-80.

Rescorla, R. A. (1978) In Cognitive Processes in Animal Behavior, H. Fowler, W. K. Honig, and S. H. Hulse, eds., pp. 15-20, L. Erlbaum Associates, Hillsdale, NJ.

Sahley, C. L., A. Gelperin, and J. W. Rudy (1981a) One-trial associative learning in a terrestrial mollusc. Proc. Natl. Acad. Sci. U. S. A. 78: 640-642.

Sahley, C. L., J. W. Rudy, and A. Gelperin (1981b) An analysis of associative learning in the terrestrial mollusc Limax maximus. I. Higher-order conditioning, blocking, and a transient US-preexposure effect. J. Comp. Physiol. A Sens. Neural Behav. Physiol. 144: 1-8.

Walters, E. T., T. J. Carew, and E. R. Kandel (1979) Associative learning in Aplysia californica. Proc. Natl. Acad. Sci. U. S. A. $76: 6675-6679$.

Walters, E. T., T. J. Carew, and E. R. Kandel (1981a) Associative learning in Aplysia: Evidence for conditioned fear in an invertebrate. Science 211: 504-506.

Walters, E. T., T. J. Carew, and E. R. Kandel (1981b) Identification of sensory neurons involved in two forms of classical conditioning in Aplysia. Soc. Neurosci. Abstr. 7: 353.

Wells, M. J. (1968) Lower Animals, McGraw-Hill, New York.

Winer, B. J. (1962) Statistical Principles in Experimental Design, McGraw-Hill, New York. 